CARDIOVASCULAR MEDICINE

\title{
Heart failure with preserved left ventricular systolic function: a hospital cohort study
}

\author{
C Berry, K Hogg, J Norrie, K Stevenson, M Brett, J McMurray
}

Heart 2005;91:907-913. doi: 10.1136/hrt.2004.041996

See end of article for authors' affiliations

.....................

Correspondence to:

Professor John J V

McMurray, Department of

Cardiology, Western

Infirmary, Glasgow G12

8QQ, UK; i.mcmurray@

bio.gla.ac.uk

Accepted

17 September 2004
Objective: To investigate how patients with heart failure with preserved left ventricular systolic function (LVSF) compare with patients with reduced LVSF.

Design: Cohort study.

Setting: Urban university hospital.

Patients: 528 index emergency admissions with heart failure during the year 2000. Information on LVSF and follow up was available for $445(84 \%)$ of these patients.

Results: 130 (29\%) patients had preserved LVSF (defined as an ejection fraction $>40 \%$ ). The median follow up was 814 days (range 632-978 days). The average (SD) age was 72 (13) years. Women accounted for $62 \%$ and $45 \%$ of patients with preserved and reduced LVSF, respectively $(p=0.001)$. Patients with preserved LVSF (compared with those with reduced LVSF) had a higher prevalence of left ventricular hypertrophy (56\% v 29\%) and aortic valve disease (mean gradient $>20 \mathrm{~mm} \mathrm{Hg} ; 31 \%$ v 9\%). Fewer patients with preserved LVSF received an angiotensin converting enzyme inhibitor $165 \%$ v $78 \%$, $\mathrm{p}=0.008)$ or spironolactone $(12 \% v 21 \%, \mathrm{p}=0.027)$. Anaemia tended to occur more often in patients with preserved LVSF than in those with reduced LVSF $(43 \%$ v 33\% for women, $p=0.12 ; 59 \%$ v $49 \%$ for men, $p=0.22$ ). There was a similarly high prevalence of significant renal dysfunction in both groups (estimated glomerular filtration rate $<60 \mathrm{ml} / \mathrm{min} / 1.73 \mathrm{~m}^{2}$ in $68 \%$ with preserved and $64 \%$ with reduced LVSF, $p=0.40)$. Mortality was similar in both groups (preserved versus reduced $51(39 \%) v 132(42 \%)$, $p=0.51)$. Compared with patients with reduced LVSF, patients with preserved LVSF tended to have a lower risk of death or hospital admission for heart failure (56 (42\%) v $165(53 \%), p=0.072$ ) but a similar rate of death or readmission for any reason.

Conclusion: Patients with preserved LVSF had more co-morbid problems than those with reduced LVSF; however, prognosis was similar for both groups.
$\mathrm{R}$ ecently, more attention has been paid to patients with heart failure (HF) who do not seem to have a major reduction in left ventricular systolic function (LVSF; usually indicated by an ejection fraction in excess of $40 \%$ or $50 \%$. Some cohort studies of hospitalised patients have suggested that up to half of all patients may have this form of HF. ${ }^{1-12}$ To date, however, most of these studies have come from the USA. ${ }^{4} 810^{11} 13$ In several, a substantial minority of patients were African American, and these were the majority in two studies. ${ }^{5}$ Furthermore, these studies also reported a very high prevalence of hypertension and patients were relatively young (the mean age in one study was 54 years). The epidemiology of HF in Europe is quite different from that in the USA in respect of ethnic background and aetiology.

Few European studies had been reported until recently. ${ }^{1214^{15}}$ One was a single hospital study from Spain $^{14}$ and another was a large national survey of French hospitals conducted in 1997. ${ }^{6}$ The French study, however, gave relatively little detail about patients with preserved LVSF. The epidemiology of HF may also differ in northern Europe. ${ }^{12}{ }^{15}$ Two studies are available from northern Europe but both are retrospective analyses of relatively old datasets and, as a consequence, describe patients receiving suboptimal treatment by current standards. Gustafsson and colleagues ${ }^{12}$ described the characteristics and outcomes of patients with HF screened for a clinical trial in 34 Danish hospitals between 1993 and 1996, whereas MacCarthy and colleagues ${ }^{15}$ described a cohort of ambulant patients with HF identified during 1993-95. Of the Danish patients, only 13\% were treated with a $\beta$ blocker and in the British cohort this proportion was $6 \%$.
Recently, the EuroHeart failure survey has reported on HF with preserved versus reduced LVSF. ${ }^{2}$ This study identified 10701 consecutive patients discharged with HF over a six week period from 115 hospitals in 24 countries of the European Society of Cardiology. Information on LVSF was available on 6806 patients. Compared with patients with reduced LVSF, patients with preserved LVSF were slightly older (by four years) and more were women (55\% v 29\%). They were also more likely to have atrial fibrillation and hypertension. The EuroHeart failure survey is one of the first studies to describe treatment differences between patients with preserved and those with reduced LVSF. ${ }^{2}$ However, other information on haematology, biochemistry (including renal function), and detailed echocardiographic findings was not provided. ${ }^{2}$ Follow up was also limited to 12 weeks. We have studied the prevalence, detailed clinical characteristics, treatment, and long term outcomes of patients with HF and preserved LVSF compared with patients with reduced LVSF in one hospital in a northern European city.

\section{METHODS}

\section{Identification of patients}

All patients discharged from the Western Infirmary, Glasgow, are supplied with an immediate discharge letter and prescription. As part of an audit of the investigation, treatment, and outcome of patients with HF, all of the

Abbreviations: $A C E$, angiotensin converting enzyme; $C O P D$, chronic obstructive pulmonary disease; eGFR, estimated glomerular filtration rate; $\mathrm{HF}$, heart failure; LVSF, left ventricular systolic function; MDRD, modification of diet in renal disease 
Table 1 Clinical characteristics of 445 patients admitted emergently with heart failure for whom left ventricular function and survival data were reported

\begin{tabular}{|c|c|c|c|c|}
\hline & $\begin{array}{l}\text { All patients } \\
(n=445)\end{array}$ & $\begin{array}{l}\text { Preserved LVSF } \\
(n=130)\end{array}$ & $\begin{array}{l}\text { Reduced LVSF } \\
(\mathrm{n}=315)\end{array}$ & $\begin{array}{l}p \text { Value (preserved } v \\
\text { reduced LVSF)* }\end{array}$ \\
\hline Men & $222(50 \%)$ & $49(38 \%)$ & $173(55 \%)$ & 0.001 \\
\hline Age (years)† & $71.8(12.8)$ & $72.6(12.4)$ & $70.5(12.9)$ & 0.11 \\
\hline \multicolumn{5}{|l|}{ Co-morbidity } \\
\hline Atrial fibrillation & $137(31 \%)$ & $48(37 \%)$ & $89(28 \%)$ & 0.074 \\
\hline Angina or MI & $165(37 \%)$ & $40(31 \%)$ & $125(40 \%)$ & 0.074 \\
\hline COPD & $44(10 \%)$ & $9(7 \%)$ & $35(11 \%)$ & 0.16 \\
\hline Diabetes & $84(30 \%)$ & $24(33 \%)$ & $60(29 \%)$ & 0.53 \\
\hline Valve disease & $21(5 \%)$ & $13(10 \%)$ & $8(3 \%)$ & 0.001 \\
\hline Anaemiał & $194(44 \%)$ & $64(49 \%)$ & $130(42 \%)$ & 0.15 \\
\hline Peptic ulcer disease & $107(24 \%)$ & $30(23 \%)$ & $79(25 \%)$ & 0.58 \\
\hline \multicolumn{5}{|l|}{ Discharge treatment } \\
\hline Loop diuretic & $387(87 \%)$ & $117(90 \%)$ & $27(86 \%)$ & 0.21 \\
\hline Thiazide diuretic & $22(5 \%)$ & $7(5 \%)$ & $15(5 \%)$ & 0.78 \\
\hline ACE inhibitor & $330(74 \%)$ & $85(65 \%)$ & $245(78 \%)$ & 0.008 \\
\hline$\beta$ Blocker & $161(36 \%)$ & $50(38 \%)$ & 111 (35\%) & 0.52 \\
\hline Spironolactone & $82(18 \%)$ & $16(12 \%)$ & $66(21 \%)$ & 0.027 \\
\hline Digoxin & $95(21 \%)$ & $30(23 \%)$ & $65(21 \%)$ & 0.57 \\
\hline Calcium channel blocker & $87(20 \%)$ & $31(24 \%)$ & $56(18 \%)$ & 0.148 \\
\hline Nitrate & $137(31 \%)$ & $35(27 \%)$ & $102(32 \%)$ & 0.25 \\
\hline Aspirin & $265(60 \%)$ & $59(46 \%)$ & $207(66 \%)$ & 0.0001 \\
\hline Warfarin & $108(24 \%)$ & $45(35 \%)$ & $63(20 \%)$ & 0.001 \\
\hline
\end{tabular}

discharge letters issued in during 2000 were reviewed for either a discharge diagnosis of HF or treatment with a combination of a diuretic and angiotensin converting enzyme (ACE) inhibitor. Case notes were reviewed for patients with a secondary diagnostic coding of HF or in whom HF was suggested by treatment with a loop diuretic/ACE inhibitor. In this case, a radiological description of pulmonary oedema on the formal chest radiograph report, in conjunction with supportive statements of typical symptoms and signs of fluid retention, was required for inclusion in this study. If these radiological and clinical features were not evident in the case record then the patient was removed from the database. Electronic death records were also searched to identify patients admitted with HF who did not survive to discharge.

Only the first emergency admission for each patient was included in this analysis. Patients with a primary or secondary diagnosis of acute myocardial infarction, identified by the presence of raised cardiac biomarkers (serum troponin $>0.2 \mu \mathrm{g} / \mathrm{l}$ or creatine kinase MB subfraction $>6 \%$ ), were not included in this analysis. Information on height and weight was inconsistently recorded in the case notes. Consequently these variables are not reported.

\section{Echocardiographic findings}

Data were obtained by a single operator on routine echocardiograms carried out for clinical reasons on admission. It is also routine practice for all departmental echocardiograms to be co-reported by cardiology clinical staff. Reduced LVSF was defined as either a left ventricular ejection fraction $<0.40$ or a qualitative report of depressed LVSF.

Haematological and biochemical measurements Haematological and biochemical measurements made at the time of admission were also analysed.

\section{Estimation of glomerular filtration rate}

Estimated glomerular filtration rate (eGFR) was calculated by an equation validated in the MDRD (modification of diet in renal disease) study as reported elsewhere ${ }^{16}$ :
$\mathrm{eGFR}=170 \times[\text { serum creatinine }]^{-0.999} \times[\text { age }]^{-0.176} \times$ [0.762 if the patient is female] $\times[1.180$ if the patient is black $] \times[\text { serum urea }]^{-0.170} \times[\text { albumin }]^{0.318}$.

\section{Follow up for death and readmission}

All patients were followed up through national electronic records as previously described from their date of admission until death or 30 September $2002 .{ }^{17}{ }^{18}$

\section{Statistical analysis}

Mean (SD) or median (interquartile range) was reported for continuous haematological, biochemical, and echocardiographic variables and number (\%) was tabulated for categorical covariates. The continuous covariates were compared between preserved and reduced LVSF with two sample $t$ tests (or Wilcoxon rank sum tests) and for the categorical factors Fisher's exact tests were used.

A Kaplan-Meier time to event curve was plotted for death and the difference between preserved and reduced LVSF was evaluated by the log rank test. Cox regression models were fitted to estimate hazard ratios (both univariate and adjusted for creatinine, anaemia, and age) for time to death (separately for in hospital (within 30 days), one year, and longer term), time to first hospital readmission for HF (with participants censored at study end or death), time to hospital readmission for any cause (with participants censored at study end or death), and the composite outcomes of time to first hospital readmission for HF or death and time to first hospital readmission for any cause or death.

To investigate whether the multivariate predictors for (a) death and (b) death or hospital readmission for HF differed between patients with preserved and those with reduced LVSF, Cox proportional hazards regression models were fitted to all the data and then, separately, to the preserved and reduced LVSF datasets individually to establish the total set of covariates univariately predictive at $\mathrm{p}<0.05$ (and with data on at least $95 \%$ of the participants). A stepwise Cox model ( $p$ to stay and $p$ to enter) was then fitted to this set of univariately predictive covariates and interaction terms between all of the univariately significant predictors and 
Table 2 Echocardiographic characteristics of patients with either preserved or reduced LVSF

\begin{tabular}{lll}
\hline & $\begin{array}{l}\text { Preserved LVSF } \\
(\mathbf{n = 1 3 0 )}\end{array}$ & $\begin{array}{l}\text { Reduced LVSF } \\
(\mathbf{n}=315)\end{array}$ \\
\hline LVEDD $(\mathrm{cm})$ & $4.7(0.6)$ & $5.8(1.0)$ \\
Left ventricular dilatation $(\mathrm{LVEDD}>5.5 \mathrm{~cm})$ & $9 / 126(7 \%)$ & $162 / 297(54 \%)$ \\
Left ventricular hypertrophy $(>1.1 \mathrm{~cm})$ & $72 / 128(56 \%)$ & $83 / 290(29 \%)$ \\
Left atrial size $(\mathrm{cm})$ & $4.6(1.1)$ & $4.4(0.7)$ \\
Left atrial dilatation $(>4.5 \mathrm{~cm})$ & $46 / 102(45 \%)$ & $94 / 206(46 \%)$ \\
Mitral regurgitation & $83 / 128(65 \%)$ & $256 / 300(85 \%)$ \\
Mitral valve forward flow velocity $(\mathrm{m} / \mathrm{s})$ & $1.27(0.52)$ & $1.04(0.37)$ \\
Mitral valve pressure half time $(\mathrm{ms})$ & $85(55)$ & $80(36)$ \\
Aortic incompetence & $37 / 128(29 \%)$ & $75 / 298(25 \%)$ \\
Aortic valve gradient (peak gradient $>20 \mathrm{~mm} \mathrm{Hg})$ & $30 / 96(31 \%)$ & $2.91(0.54)$ \\
Tricuspid valve regurgitation peak velocity $(\mathrm{m} / \mathrm{s})$ & $2.90(0.49)$ & $40(13)$ \\
Estimated pulmonary artery pressure (mm Hg) & $40(12)$ & \\
\hline *Data are proportions of patients with a measurement of the relevant variable or mean $(S D)$ for continuous \\
variables. \\
LVEDD, left ventricular end diastolic diameter.
\end{tabular}

LVSF were individually tested for significance in the presence of the covariates identified in the stepwise model. Separate models for reduced and preserved function adjusting for all the stepwise covariates and all covariates with significant interactions with LVSF were then reported.

A result with $\mathrm{p}<0.05$ was taken as being significant. No adjustment has been made for multiple comparisons. All statistical analyses were undertaken with Stata version 7 software (StataCorp LP, College Station, Texas, USA) and SAS 8.2 for Windows (SAS Institute, Cary, North Carolina, USA).

\section{RESULTS}

\section{Baseline clinical characteristics}

Of 528 patients with a discharge diagnosis of HF, 445 had a report of left ventricular function and follow up information. All subsequent analyses refer to this subset of patients. Table 1 lists their clinical characteristics. One hundred and thirty (29\%) patients had preserved LVSF. More of them were women compared with the group of patients with reduced LVSF $(62 \% v 45 \%, \mathrm{p}=0.001)$. Patients with reduced LVSF were more likely to have a history of angina or myocardial infarction, whereas those with preserved LVSF were more likely to have atrial fibrillation or valve disease. One hundred and twenty one $(27 \%)$ patients had no other concomitant diagnosis (for example, no atrial fibrillation, valve disease, chronic obstructive pulmonary disease (COPD), or anaemia). Twenty four (18\%) patients with preserved LVSF and 97 (31\%) patients with reduced LVSF had no other co-morbid diagnosis $(\mathrm{p}=0.003)$.

\section{Medical treatment}

The proportion of patients treated with a loop diuretic was similar in the two groups as was the mean dose of furosemide (82 (93) $\mathrm{mg}$ in the preserved LVSF group compared with 89 (102) $\mathrm{mg}$ in the reduced LVSF group). Patients with preserved LVSF were treated with an ACE inhibitor less often than those with reduced LVSF (table 1). This was also the case for spironolactone. There was lower use of aspirin but higher use of warfarin among patients with preserved LVSF compared with those with reduced LVSF (table 1).

\section{Echocardiography}

Compared with patients with reduced LVSF, a higher proportion of patients with preserved LVSF had left ventricular hypertrophy but a smaller proportion had left

Table 3 Baseline haematological and biochemical findings according to preserved or reduced LVSF

\begin{tabular}{|c|c|c|c|c|}
\hline & All $(n=442)^{*}$ & $\begin{array}{l}\text { Preserved LVSF } \\
(n=130)\end{array}$ & $\begin{array}{l}\text { Reduced LVSF } \\
(n=315)\end{array}$ & $\begin{array}{l}\mathrm{p} \text { Value (preserved } v \\
\text { reduced LVSF) } \dagger\end{array}$ \\
\hline \multicolumn{5}{|l|}{ Haemoglobin (g/l) } \\
\hline Men & $126(21)$ & $121(21)$ & $127(21)$ & 0.088 \\
\hline Women & $120(19)$ & 116 (22) & $122(17)$ & 0.046 \\
\hline \multicolumn{5}{|l|}{ Packed cell volume } \\
\hline Men & $0.384(0.058)$ & $0.371(0.060)$ & $0.388(0.056)$ & 0.076 \\
\hline Women & $0.370(0.055)$ & $0.358(0.059)$ & $0.377(0.052)$ & 0.0238 \\
\hline$M C V(f)$ & $90.2(6.7)$ & $90.0(7.5)$ & $89.9(6.8)$ & 0.88 \\
\hline Sodium (mmol/l) & $139.4(4.1)$ & $139.5(3.8)$ & $139.3(4.3)$ & 0.72 \\
\hline Potassium (mmol//) & $4.2(0.6)$ & $4.2(0.7)$ & $4.3(0.6)$ & 0.48 \\
\hline Urea (mmol/l) & $10.6(7.6)$ & $10.4(6.7)$ & $10.6(8.0)$ & 0.76 \\
\hline Creatinine $(\mu \mathrm{mol} / \mathrm{l})$ & $134(89)$ & $126(66)$ & $137(96)$ & 0.17 \\
\hline \multicolumn{5}{|l|}{ eGFR $\left(1 / \mathrm{min} / 1.73 \mathrm{~m}^{2}\right) \ddagger$} \\
\hline$>90$ & $17(4 \%)$ & $3(3 \%)$ & $14(5 \%)$ & 0.47 \\
\hline $60-90$ & $121(31 \%)$ & $34(29 \%)$ & $87(31 \%)$ & \\
\hline $30-60$ & $191(49 \%)$ & $62(54 \%)$ & $129(46 \%)$ & \\
\hline$<30$ & $65(16 \%)$ & $17(15 \%)$ & $48(17 \%)$ & \\
\hline Glucose (mmol/l) & $8.9(17.8)$ & $7.9(3.7)$ & $9.4(21.1)$ & 0.28 \\
\hline Albumin (mmol/li) & $38.0(4.5)$ & $38.5(4.4)$ & $37.8(4.5)$ & 0.12 \\
\hline
\end{tabular}

All values are given as mean (SD)

*Haemoglobin and packed cell volume data were missing for three patients: one patient had preserved LVSF and two had reduced LVSF; †two sample $t$ test; łcalculable for 394 patients, of whom 116 (29\%) had preserved LVSF and $278(70 \%)$ had reduced LVSF.

eGFR, estimated glomerular filtration rate; MCV, mean corpuscular volume. 


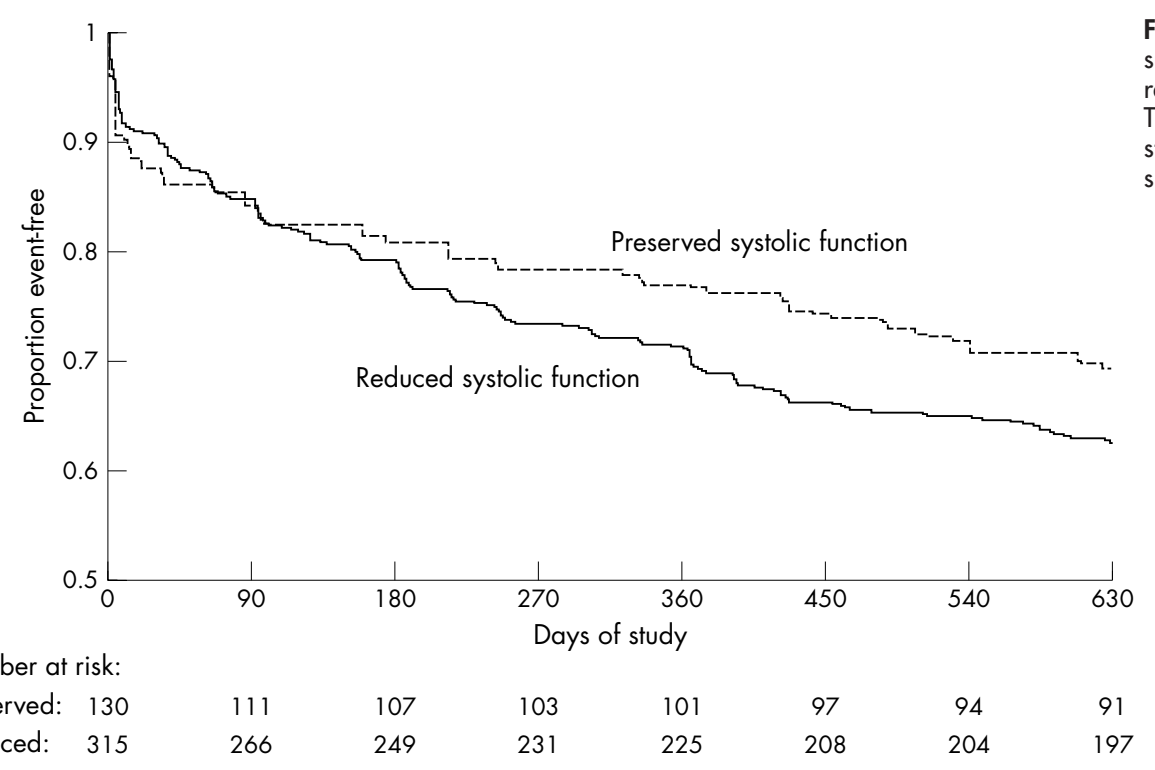

Figure 1 Proportion with event-free survival of patients with preserved or reduced leff ventricular systolic function. The numbers of patients at risk at the start of each period of 90 days are shown.

Number at risk:

Preserved: 130

266

249

231

225

$208 \quad 204$

197

ventricular dilatation (table 2). Fewer patients with preserved LVSF had mitral regurgitation (65\% v 85\%). Mitral valve forward flow velocity was greater in patients with preserved LVSF than in those with reduced LVSF.

\section{Haematology}

Both men and women with preserved LVSF had lower haemoglobin concentrations than men and women with reduced LVSF (table 3 ).

\section{Biochemistry}

The proportions of patients with an increased serum creatinine concentration and reduced eGFR were similar in patients with preserved LVSF and with reduced LVSF (table 3).

\section{Clinical outcomes}

The median follow up was 814 days (range 632-978 days).

\section{Duration of index hospital admission}

Mean (SD) duration of admission was 7 (7) days for patients with preserved LVSF compared with 7 (6) days for those with reduced LVSF $(p=0.99)$.

\section{Death}

Sixteen $(12 \%)$ patients with preserved LVSF and $30(10 \%)$ patients with reduced LVSF died in hospital (log rank $\mathrm{p}=0.38$; after adjustment for other prognostic covariates (age $>65$ years, renal dysfunction (eGFR $<60 \mathrm{ml} / \mathrm{min} /$ $1.73 \mathrm{~m}^{2}$ ), and anaemia) $\mathrm{p}=0.63$ ).

Figure 1 shows the Kaplan-Meier survival estimates for patients with preserved and reduced LVSF. By one year, 29 (22\%) patients with preserved LVSF had died compared with $93(30 \%)$ of those with reduced LVSF ( $\log$ rank $\mathrm{p}=0.16$; after covariate adjustment, $\mathrm{p}=0.13$ ). Survival was similar in the two groups over the whole duration of follow up-that is, $51(39 \%)$ died in the preserved LVSF group compared with $132(42 \%)$ in the reduced LVSF group ( $\log$ rank $p=0.51$; after covariate adjustment, $\mathrm{p}=0.26$ ).

\section{Hospital readmission}

Patients with preserved LVSF had a lower rate of readmission for HF than patients with reduced LVSF (14 (11\%) v 55 $(17 \%), p=0.054$; after covariate adjustment, $\mathrm{p}=0.13$ ) but a similar rate of subsequent admission for any reason (95 $(73 \%)$ v $233(74 \%), p=0.72$; after covariate adjustment, $\mathrm{p}=0.58)$.

\section{Death or readmission}

Patients with preserved LVSF had similar rates of death or readmission for any reason to those with reduced LVSF ( 115 $(88 \%)$ v 277 (88\%), p = 0.90; after covariate (anaemia, renal dysfunction, and age $>65$ years) adjustment, $p=0.63$ ). Patients with preserved LVSF had a lower risk of death or hospital admission for HF (56 (42\%) v 165 (53\%), p = 0.070; adjusted $\mathrm{p}=0.072$ ).

\section{Predictors of death and death or hospital readmission for HF}

The set of covariates that predicted death in both the preserved and reduced LVSF groups were age, sex, haemoglobin, neutrophil count, presence of valve disease, treatment with spironolactone, and plasma concentrations of sodium and urea (table 4). In addition, we found that COPD significantly interacted with LVSF. COPD was highly predictive of death in the preserved LVSF group but not in the reduced LVSF group. We found the same set of predictors for the outcome of death or hospital readmission for HF. However, in addition to COPD, male sex was predictive in the preserved, but not the reduced, LVSF group. There was also a significant interaction between urea and LVSF, whereby urea was a stronger predictor in the preserved group (hazard ratio 1.81 ) than in the reduced group (hazard ratio 1.18), although urea was still a significant predictor in the reduced LVSF group.

\section{DISCUSSION}

This study confirms the findings of prior reports and provides new information on this interesting and neglected type of HF. We observed that the prognosis of patients with HF and preserved LVSF is comparable with that of patients with reduced LVSF. The predictors of adverse outcome, however, differ between the two groups.

The proportion of patients in our study who had HF and preserved LVSF was $29 \%$, consistent with the range of 24$55 \%$ in similar studies from the USA. ${ }^{4578101113}$ The proportion in the one Japanese study we know of was $35 \% .{ }^{19}$ In the EuroHeart failure survey $46 \%$ of participants with a measurement of LVSF had preserved systolic function, 
Table 4 Predictors of (a) death and (b) death or readmission to hospital for heart failure and for preserved and reduced LVSF from a multivariate Cox proportional hazards regression model

\begin{tabular}{|c|c|c|c|c|c|}
\hline \multirow[b]{2}{*}{ Factor } & \multirow{2}{*}{$\begin{array}{l}\text { Incremental } \\
\text { factor }\end{array}$} & \multicolumn{2}{|l|}{ Preserved LVSF } & \multicolumn{2}{|l|}{ Reduced LVSF } \\
\hline & & HR $(95 \% \mathrm{Cl})$ & $\mathrm{p}$ Value & HR $(95 \% \mathrm{Cl})$ & $\mathrm{p}$ Value \\
\hline \multicolumn{6}{|l|}{ (a) Death } \\
\hline Age & 5 years & $1.17(0.99$ to 1.37$)$ & 0.062 & 1.18 (1.08 to 1.30$)$ & 0.0004 \\
\hline Haemoglobin & $20 \mathrm{~g} / \mathrm{l}$ & $0.71(0.52$ to 0.96$)$ & 0.024 & $0.69(0.56$ to 0.84$)$ & 0.0003 \\
\hline Sodium & $5 \mathrm{mmol} / \mathrm{l}$ & $0.69(0.48$ to 0.98$)$ & 0.035 & $0.82(0.67$ to 1.00$)$ & 0.052 \\
\hline Neutrophils & $3 \times 10^{9} / 1$ & $1.10(0.88$ to 1.37$)$ & 0.40 & $1.32(1.19$ to 1.47$)$ & $<0.0001$ \\
\hline Urea & $5 \mathrm{mmol} / \mathrm{l}$ & $1.53(1.25$ to 1.87$)$ & $<0.0001$ & $1.26(1.14$ to 1.38$)$ & $<0.0001$ \\
\hline Spironolactone & Yes & 1.23 (0.56 to 2.72$)$ & 0.61 & 1.87 (1.23 to 2.83 ) & 0.0032 \\
\hline Valve disease & Yes & $5.82(2.43$ to 14.0$)$ & $<0.0001$ & $2.82(1.10$ to 7.24$)$ & 0.032 \\
\hline Sex & Male & 2.44 (1.25 to 4.79$)$ & 0.0094 & $0.96(0.67$ to 1.38$)$ & 0.83 \\
\hline \multicolumn{6}{|c|}{ Covariate with significant interactions with systolic function } \\
\hline COPD & Y & 11.6 (5.11 to 26.2$)$ & $<0.0001$ & $1.64(0.94$ to 2.88$)$ & 0.084 \\
\hline \multicolumn{6}{|c|}{$\begin{array}{l}\text { Tests for interaction: COPD } p=0.0006 \\
\text { (b) Death or hospital readmission for HF }\end{array}$} \\
\hline Age & 5 years & $1.06(0.92$ to 1.22$)$ & 0.39 & $1.12(1.04$ to 1.21$)$ & 0.0030 \\
\hline Haemoglobin & $20 \mathrm{~g} / \mathrm{l}$ & $0.83(0.62$ to 1.12$)$ & 0.22 & $0.73(0.61$ to 0.87$)$ & 0.0006 \\
\hline Sodium & $5 \mathrm{mmol} / \mathrm{l}$ & $0.60(0.43$ to 0.84$)$ & 0.0030 & $0.92(0.76$ to 1.12$)$ & 0.42 \\
\hline Neutrophils & $3 \times 10^{9} / 1$ & $1.17(0.94$ to 1.45$)$ & 0.15 & $1.25(1.12$ to 1.40$)$ & 0.0001 \\
\hline Spironolactone & Yes & 1.39 (0.65 to 2.94$)$ & 0.40 & 1.96 (1.35 to 2.85 ) & 0.0004 \\
\hline Valve disease & Yes & 3.96 (1.67 to 9.38$)$ & 0.0018 & $3.72(1.68$ to 8.24$)$ & 0.0012 \\
\hline \multicolumn{6}{|c|}{ Covariates with significant interactions with systolic function: } \\
\hline COPD & Yes & $9.86(4.48$ to 21.7$)$ & $<0.0001$ & 1.61 (0.98 to 2.64$)$ & 0.061 \\
\hline Sex & Male & $1.69(0.91$ to 3.15$)$ & 0.038 & $0.84(0.60$ to 1.16$)$ & 0.29 \\
\hline Urea & $5 \mathrm{mmol} / \mathrm{l}$ & 1.81 (1.46 to 2.24$)$ & $<0.0001$ & 1.18 (1.07 to 1.29$)$ & 0.0006 \\
\hline \multicolumn{6}{|c|}{ Tests for interaction: COPD $p=0.0002$, sex $p=0.046$, urea $p=0.0004$} \\
\hline
\end{tabular}

though only $64 \%$ of patients had such a measurement. ${ }^{2}$ In our study the average age of patients with HF and preserved LVSF was 72.6 years, a little older than that of patients with reduced LVSF (mean age 70.5 years, $p=0.11$ ). Compared with patients with reduced LVSF, more patients with a preserved LVSF tended to be older than 65 years ( $80 \% v 72 \%$, $\mathrm{p}=0.093)$. This too is consistent with prior studies where patients with preserved LVSF were older, though in only three studies were patients as old as ours. ${ }^{6710}$ Similarly, the proportion of women with preserved LVSF (62\%) was comparable with previous reports.

The present report also adds new information to the growing literature on HF with preserved LVSF in a number of important ways. In earlier studies, detailed information on the prevalence and nature of co-morbid problems, such as valvar heart disease and anaemia, and their relations with outcome are not reported.246791011 1415 Furthermore, none of these reports provided a detailed description of renal function. Surprisingly, most studies also did not provide information on cardiac anatomy (determined echocardiographically).

The few existing studies describing renal function reported serum creatinine concentration, which can be an inaccurate measure, especially in frail elderly patients. We were able to calculate eGFR of our patients by using a validated formula. The majority of patients had a significant reduction in eGFR and, interestingly, the proportions with important reductions in eGFR were comparable in the two types of HF.

We also examined the prevalence of anaemia in our cohort, in view of the new interest in this co-morbidity as an independent predictor of worse outcome and because correcting anaemia may improve symptoms and outcome. We found a high overall prevalence of anaemia in our patients. Anaemia tended to be more common in patients with preserved LVSF than in those with reduced LVSF. We know of no prior report of the frequency of anaemia in a study of patients with HF that took account of ventricular function.
The most probable explanation for the HF syndrome in patients with preserved LVSF is diastolic dysfunction. The most probable substrate for this is left ventricular hypertrophy. We identified left ventricular hypertrophy (by echocardiography) in $56 \%$ of patients with preserved LVSF compared with $29 \%$ of patients with reduced LVSF. This observation is a much larger differential than in any of the prior hospital cohort studies. These earlier studies, however, generally reported ECG left ventricular hypertrophy. We also found that left atrial enlargement is as common in patients with preserved LVSF as in those with reduced LVSF. This was despite mitral regurgitation occurring more commonly in those with reduced LVSF. Furthermore, mitral valve forward flow velocity was greater in patients with preserved LVSF. Taken together, these findings suggest that left atrial pressure may have been greater in patients with preserved LVSF than in those with reduced LVSF.

We found that patients with preserved LVSF had a similar in-hospital length of stay to those with reduced LVSF, in keeping with other European ${ }^{2}{ }^{6}$ and US studies. ${ }^{3}{ }^{20}$

Very few studies have described inpatient case fatality rates. Philbin and colleagues ${ }^{20}$ reported a $3 \%$ rate in patients with preserved compared with a 5\% rate in those with reduced LVSF in New York State. Cohen-Solal and colleagues, ${ }^{6}$ in a national survey of French hospitals, found these rates to be $3 \%$ and $8 \%$, respectively. Ours were $12 \%$ and $10 \%$, respectively. In other words, all three studies show that patients with preserved LVSF have a similar short term outcome.

Longer term mortality, time to first hospital readmission for any cause or for HF, and time to the composite outcome of readmission for any reason or death were similar in our patients with preserved or with reduced LVSF. We did find that the time to first readmission for HF or death was lower in patients with preserved LVSF than in patients with reduced LVSF. Overall, these findings are in keeping with the results of some f $^{10152122}$ but not all studies 9112324 . The differences in findings between these studies and our own 
may be explained by differences in the type of population studied, such as hospitalised versus ambulatory patients, ${ }^{15}$ co-morbidity, age, ${ }^{10}$ and duration of follow up. ${ }^{7}$

Common predictors of adverse outcome in patients with preserved or reduced LVSF were age, haemoglobin, neutrophil count, the presence of valve disease, treatment with spironolactone, and plasma concentrations of sodium and urea. Although most of these prognostic markers have been previously described, this is the first report of the prevalence and prognostic importance of haemoglobin in patients with HF and preserved LVSF. Furthermore, important differences were observed between patients with reduced and patients with preserved LVSF in factors that predict outcome. For example, COPD and male sex predicted mortality in patients with preserved LVSF but not in those with reduced LVSF. We have also described for the first time a quantitative interaction of renal function with LVSF, with serum urea concentration being a much stronger predictor of death or readmission for HF in patients with preserved LVSF than in those with reduced LVSF. Our findings underline the importance of other health problems in patients with preserved LVSF and are consistent with the notion that these patients are less likely to die of a cardiac problem and more likely to die of a non-cardiovascular cause in the long term. ${ }^{25}$

Lastly, we looked at treatment. Our overall rates of use of ACE inhibitors and, especially, $\beta$ blockers are much greater than in prior reports, reflecting the more contemporary nature of our study. We found higher rates of use of ACE inhibitors and spironolactone in patients with reduced LVSF than in those with preserved LVSF The differential in the use of ACE inhibitors is in keeping with previous studies and is expected. The relatively low rates of spironolactone use are also in keeping with the results of the EuroHeart failure survey. ${ }^{2}$ Compared with other studies, we observed a greater overall use of $\beta$ blockers than in some earlier reports ${ }^{6}{ }^{12}$ but a similar rate of $\beta$ blocker use to that in one more contemporary North American population. ${ }^{7}$ We also found similar rates of use of $\beta$ blockers in the patients with preserved and reduced LVSF. Prior findings with $\beta$ blockers, according to LVSF, have been less consistent, with two studies reporting higher rates of use in patients with preserved LVSF. There was a considerably greater use of warfarin in patients with preserved LVSF, in keeping with the higher prevalence of atrial fibrillation in these patients. Atrial fibrillation is an important risk factor for $\mathrm{HF}$ and is an adverse prognostic marker when HF is present. ${ }^{26}$ That atrial fibrillation is more prevalent in patients with preserved LVSF suggests that it is an important co-morbid problem in these patients. Aspirin use was greater in patients with reduced LVSF, in keeping with the greater evidence of coronary heart disease in this group.

As with any study of this type there are a number of limitations. One is generic to most studies of this type: the difficulty in assuring a diagnosis of HF in patients with preserved LVSF in the absence of an ideal test for this condition. In the future natriuretic peptides may help. A second limitation is that we chose to exclude patients with a diagnosis of myocardial infarction on the basis of increased cardiac biomarkers. There has been conflicting opinion on whether patients with a primary diagnosis of myocardial infarction and secondary HF should be included in a study of HF. The issue has become even more clouded by the recognition that troponin can be increased in a minority of patients with acutely decompensated HF without a clinically recognisable myocardial infarction.

In conclusion, there were many similarities and differences between patients with preserved LVSF and those with reduced LVSF. Patients with preserved LVSF had more co- morbidity including, notably, anaemia. Both groups have a similar and greatly reduced level of renal function. Despite quite distinctly different echocardiographic findings and treatment, patients with preserved LVSF had similar long term risks of death or readmission to hospital to those with reduced LVSF. Sex, COPD, and renal function are stronger predictors of adverse outcome in patients with preserved LVSF than in patients with reduced LVSF.

\section{ACKNOWLEDGEMENTS}

We thank Susan Frame and Bev Dodds of the Information and Statistics Division, Common Services Agency, Edinburgh.

\section{Authors' affiliations}

C Berry, K Hogg, K Stevenson, M Brett, J McMurray, Department of Cardiology, Western Infirmary, Glasgow, UK

J Norrie, Centre for Healthcare Randomised Trials (CHaRT), Health

Services Research Unit, Aberdeen University, Aberdeen, UK

\section{REFERENCES}

1 Gottdiener JS, McClelland RL, Marshall R, et al. Outcome of congestive heart failure in elderly persons: influence of left ventricular systolic function. The cardiovascular health study. Ann Intern Med 2002;137:631-9.

2 Lenzen MJ, Scholte op Reimer WJM, Boersma E, et al. Differences between patients with a preserved and a depressed left ventricular function: a report from the EuroHeart failure survey. Eur Heart J 2004;25:1214-20.

3 Lora JS, Porras JJ, Pedrajas JNA, et al. Heart failure in internal medicine departments. Med Clin 2002;1 18:605-10.

4 Malki Q, Sharma ND, Afzal A, et al. Clinical presentation, hospital length of stay, and readmission rate in patients with heart failure with preserved and decreased left ventricular systolic function. Clin Cardiol 2002:25:149-52.

5 Philbin EF, McCullough PA, Dec GW, et al. Length of stay and procedure utilization are the major determinants of hospital charges for heart failure. Clin Cardiol 2001;24:56-62

6 Cohen-Solal A, Desnos M, Delahaye F, et al. A national survey of heart failure in French hospitals. Eur Heart J 2000;21:763-9.

7 Smith GL, Masoudi FA, Vaccarino V, et al. Outcomes in heart failure patients with preserved ejection fraction: mortality, readmission, and functional decline. J Am Coll Cardiol 2003;41:1510-8

8 Philbin EF, DiSalvo TG. Influence of race and gender on care process, resource use, and hospital-based outcomes in congestive heart failure. Am J Cardiol 1998;82:76-81.

$9 \mathrm{McDermott}$ MM, Feinglass J, Lee Pl, et al. Systolic function, readmission rates, and survival among consecutively hospitalized patients with congestive heart failure. Am Heart J 1997; 134:728-36.

10 Pernenkil R, Vinson JM, Shah AS, et al. Course and prognosis in patients $>=70$ years of age with congestive heart failure and normal versus abnormal left ventricular ejection fraction. Am J Cardiol 1997;79:216-9.

11 Feinglass J, Martin GJ, Lin E, et al. Is heart failure survival improving? Evidence from 2323 elderly patients hospitalized between 1989-2000. Am Heart J 2003;146:111-4.

12 Gustafsson F, Torp-Pedersen C, Brendorp B, et al. Long-term survival in patients hospitalized with congestive heart failure: relation to preserved and reduced systolic function. Eur Heart J 2003;24:863-70.

13 Vaccarino V, Gahbauer E, Kasl SV, et al. Differences between African Americans and whites in the outcome of heart failure: evidence for a greater functional decline in African Americans. Am Heart J 2002;143:1058-67.

14 Miralda GP, Soriano N, Brotons C, et al. Baseline characteristics and determinants of outcome in a patient population admitted for heart failure to a general hospital. Rev Esp Cardiol 2002;55:571-8.

15 MacCarthy PA, Kearney MT, Nolan J, et al. Prognosis in heart failure with preserved left ventricular systolic function: prospective cohort study. BMJ 2003;327:78-9.

16 Levey AS, Bosch JP, Lewis JB, et al. A more accurate method to estimate glomerular filtration rate from serum creatinine: a new prediction equation. Ann Intern Med 1999;330:877-84

17 Maclntyre K, Capewell S, Stewart S, et al. Evidence of improving prognosis in heart failure: trends in case fatality in 66547 patients hospitalized between 1986 and 1995. Circulation 2000;102:1126-31.

18 Stewart S, Maclntyre K, MacLeod MMC, et al. Trends in hospitalization for heart failure in Scotland, 1990-1996: an epidemic that has reached its peak? Eur Heart J 2001;22:209-17.

19 Tsuchihashi M, Tsutsui H, Kodama K, et al. Clinical characteristics and prognosis of hospitalized patients with congestive heart failure: a study in Fukuoka, Japan. Jpn Circ J 2000;64:953-9.

20 Philbin EF, Rocco TA, Lindenmuth NW, et al. Systolic versus diastolic heart failure in community practice: clinical features, outcomes, and the use of angiotensin-converting enzyme inhibitors. Am J Med 2000;109:605-13

21 Luchi RJ, Snow E, Luchi JM, et al. Left ventricular function in hospitalized geriatric patients. J Am Geriatr Soc 1982;30:700-5. 
22 Kinney EL, Wright RJ. Survival in patients with heart failure and normal basal systolic wall motion. Angiology 1989;40:1025-9.

23 Kupari $M$, Lindroos $M$, livanainen $A M$, et al. Congestive heart failure in old age: Prevalence, mechanisms and 4-year prognosis in the Helsinki Ageing Study. J Int Med 1997:241:387-94.

24 Ansari $M$, Alexander $M$, Tutar $A$, et al. Incident cases of heart failure in a community cohort: importance and outcomes of patients with preserved systolic function. Am Heart J 2003;146:115-20.
25 Curtis JP, Sokol SI, Wang $Y$, et al. The association of left ventricular ejection fraction, mortality, and cause of death in stable outpatients with heart failure. J Am Coll Cardiol 2003;42:736-42.

26 Dries DL, Exner DV, Gersh BJ, et al. Atrial fibrillation is associated with an increased risk for mortality and heart failure progression in patients with asymptomatic and symptomatic left ventricular systolic dysfunction: a retrospective analysis of the SOLVD trials. J Am Coll Cardiol 1998;32:695-703.

\section{IMAGES IN CARDIOLOGY}

An abnormal ECG with inverted T waves in the precordial leads: confirming the diagnosis with contrast enhanced echocardiography

\begin{abstract}
A 68 year old woman was referred to our cardiology department for a preoperative evaluation after the finding of a systolic ejection murmur and an abnormal ECG. She had a history of diabetes mellitus, hypertension, and hypercholesterolaemia. She denied any history of chest pain, palpitations, or dyspnoea on exertion. Except for a raised blood pressure of $165 / 70 \mathrm{~mm} \mathrm{Hg}$ and a systolic murmur with the punctum maximum at the second right intercostal space, physical examination revealed no further abnormalities. The ECG showed a sinus rhythm with T wave inversion in the inferior and anterolateral leads with giant negative T waves in leads V3-V6 (panel A). The echocardiogram raised the suspicion of a hypertrophic cardiomyopathy (HCM); however, this could not be demonstrated with fundamental echocardiography (panel B). Transthoracic contrast enhanced echocardiography demonstrated the diagnosis of apical HCM, showing a typical spade-like lumen of the left ventricle (panel C). Magnetic resonance imaging confirmed the myocardial thickening of the apex of the left ventricle (panel D).

Although more common in Japan, apical HCM is a relatively rare form of hypertrophic cardiomyopathy in western countries. Giant negative $\mathrm{T}$ waves on the ECG in asymptomatic patients should raise the suspicion of apical HCM. As contrast enhanced echocardiography improves visualisation of the myocardial wall, administration of an ultrasound contrast agent in patients with these typical ECG abnormalities is recommended, especially in patients with suboptimal and/or non-diagnostic windows.
\end{abstract}

\section{P A Dijkmans C A Visser O Kamp pa.dijkmans@vumc.nl}

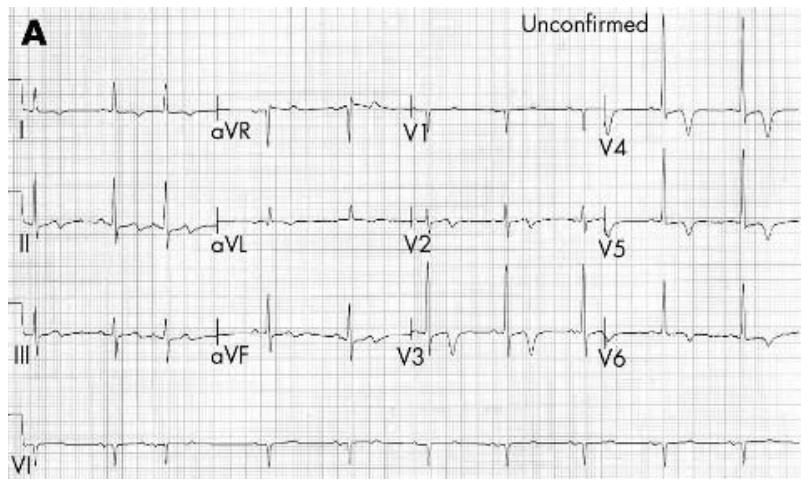

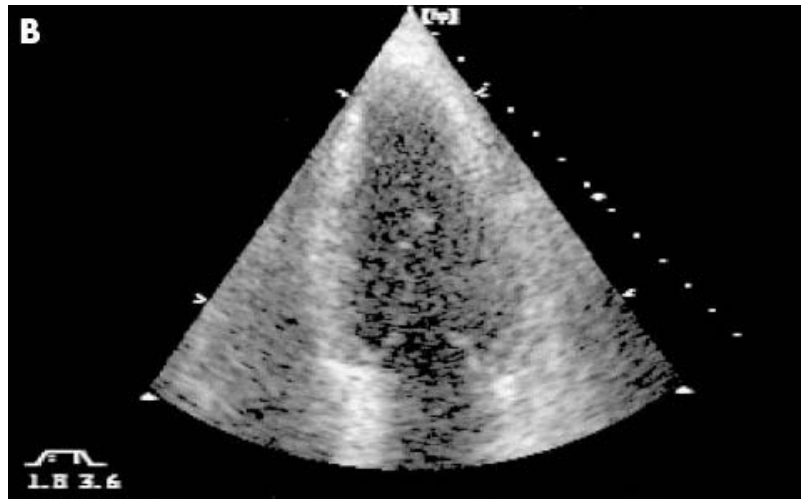
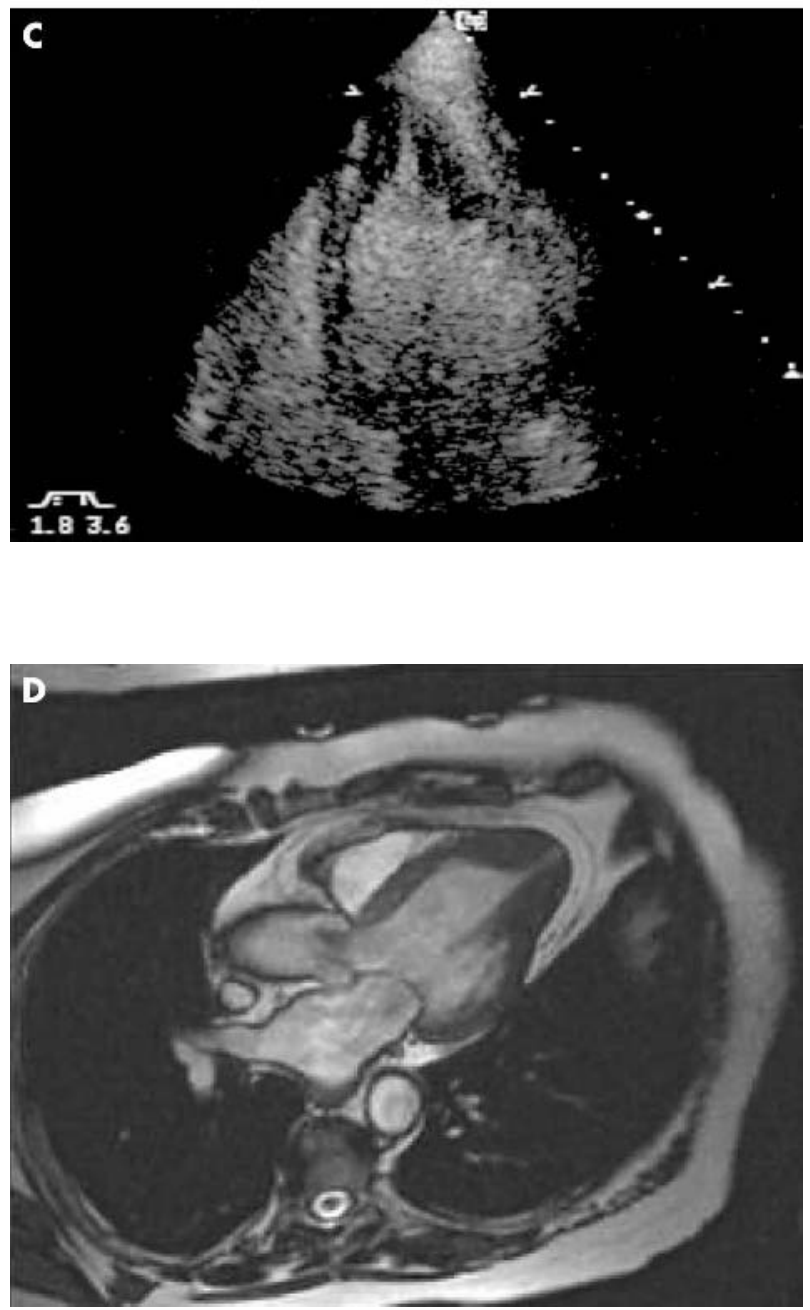